\title{
El modelo social de discapacidad en el nivel universitario: supuestos, implicancias, avances y desafíos
}

\section{The social model of disability at the university level: assumptions, implications, progress and challenges}

ARTÍCULO

\author{
Mariana Luzuriaga \\ Universidad de San Andrés, Argentina. Contacto: mluzuriaga@udesa.edu.ar
}

Recibido: agosto de 2020

Aceptado: octubre de 2020

\section{Resumen}

En las últimas décadas el Modelo Social de discapacidad cobró relevancia en la orientación de políticas de inclusión educativa en el nivel universitario argentino. Bajo el supuesto de que las causas de la discapacidad son fundamentalmente sociales, pone el foco en las acciones necesarias para superar las barreras que obturan garantizar el derecho a la educación de las personas discapacitadas "en igualdad de condiciones" que las demás. Pero la significación de esta proclama, cuya concreción está lejos de ser alcanzada, exige analizar detenidamente sus supuestos e implicancias desde una perspectiva filosófico-política. Entendiendo que la discapacidad constituye una "comunidad bivalente" (Fraser, 2000), en el presente trabajo argumentaremos que solo en la medida en que se traduzca en políticas que se sustenten mancomunadamente en la redistribución y el reconocimiento el Modelo Social presenta una orientación fértil para la justicia educativa. Para ello se examinará la naturaleza de los factores de exclusión históricamente heredados y de algunas políticas implementadas en respuesta a nivel institucional, tomando como ejes de análisis la "accesibilidad formal", la "accesibilidad académica" y la "accesibilidad psico-socio-cultural". Concluimos señalando algunos desafíos pendientes y posibles implicancias de trabajos como este.

Palabras clave: modelo social de discapacidad; justicia social; redistribución; reconocimiento; nivel universitario.

\section{Abstract}

Over the last decades, the Social Model of Disability gained relevance in the orientation of educational inclusion policies at the university level in Argentina. Under the assumption that 
the causes of disability are fundamentally social, it focuses on the actions necessary to overcome the barriers that undermine disabled people's right to education "on equal terms" as their peers. But the significance of this proclamation, whose concretion is far from being achieved, requires a careful analysis of its assumptions and implications from a philosophical-political perspective. Understanding that disability constitutes a "bivalent community" (Fraser, 2000), in the present work we will argue that only insofar as it is translated into policies that are jointly supported by redistribution and recognition, does the Social Model present a fertile orientation for educational justice. For this, the nature of the historically inherited exclusion factors and of some policies implemented in response at the institutional level will be examined, considering three categories of analysis: "formal accessibility", "academic accessibility" and "psycho-socio-cultural accessibility". We conclude by pointing out some pending challenges and possible implications of articles like this one.

Keywords: social model of disability; social justice; redistribution; recognition; university level.

\section{Introducción}

En Argentina, como en otras partes del mundo, en los últimos años el llamado "Modelo Social de discapacidad" ha cobrado creciente importancia como paradigma para la orientación de políticas de inclusión social en general, incluyendo la inclusión educativa", foco central del presente trabajo. Bajo el supuesto de que las causas de la discapacidad son fundamentalmente sociales y culturales, este modelo pone el foco en la responsabilidad social por las acciones necesarias para superar las barreras que obturan el derecho a la educación de las personas discapacitadas "en igualdad de condiciones" que las demás. Mientras a priori este podría ser un enfoque prometedor, cuyo atractivo discursivo lo vuelve difícilmente objetable, la significación de proclamas como "inclusión", "responsabilidad social" e "igualdad de condiciones" exige analizar detenidamente sus supuestos e implicancias desde una perspectiva filosófico-política.

El debate filosófico sobre la justicia educativa a partir de Rawls (1971) ha girado en gran medida en torno del ideal de igualdad de oportunidades (Fishkin, 1983). Por mucho tiempo, este debate fue abordado mayormente desde el paradigma de la "justicia distributiva”, por lo que el foco estuvo puesto en la compensación de las desigualdades que tienen un origen socioeconómico (riqueza, ingresos). Sin embargo, la "desestabilización" de

\footnotetext{
${ }^{1}$ Aquí como a lo largo de todo el trabajo se utiliza una acepción restringida de la "inclusión educativa", vinculada específicamente a la discapacidad.
} 
dicho ideal y el avance de las reivindicaciones provenientes de la "política de la identidad" y el "reconocimiento de la diferencia" (Fraser y Honneth, 2005) generaron la necesidad de ampliar la comprensión de las políticas de igualación de oportunidades (Bolívar, 2012).

En el marco de esta mutación del debate sobre la justicia social en general y sobre la justicia educativa en particular, este trabajo se inserta en el corpus de literatura que discute las distinciones de paradigmas y dimensiones de la justicia que operan como marcos teóricos y programáticos de las proclamas antes citadas. En particular, retomando la concepción bidimensional de la justicia social de Nancy Fraser (2000) y los desarrollos de Iris Marion Young en La justicia y la política de la diferencia, los objetivos del presente trabajo son:

Objetivo general: Argumentar que solo en la medida en que el Modelo Social de discapacidad se traduzca en políticas que se sustenten mancomunadamente en la redistribución y el reconocimiento presenta un modelo fértil para la justicia educativa.

Objetivo específico: Analizar los supuestos de justicia educativa implicados en las autodefinidas políticas de inclusión educativa vinculadas a la discapacidad en el nivel universitario argentino.

A fin de exponer cómo se conjugan las dimensiones de la justicia en dichas políticas, se tomarán como ejes de análisis tres categorías usualmente utilizadas en la literatura y los marcos normativos: (i) la accesibilidad formal, (ii) la accesibilidad académica, y (iii) la accesibilidad psico-socio-cultural. En cada caso se ahondará en sus definiciones e implicancias. Explicitando cuáles son los factores de exclusión históricamente heredados, se examinarán los supuestos vinculados a cada paradigma de justicia educativa. Además, se citarán algunos ejemplos ilustrativos de cómo se les ha dado respuesta a nivel institucional en universidades nacionales del Área Metropolitana de Buenos Aires, retomando los resultados de un estudio empírico previo (Luzuriaga, 2015).

El trabajo está estructurado de la siguiente manera. Comenzaremos situando históricamente el surgimiento del Modelo Social de discapacidad puesto que, si bien nuestro interés es comprender la coyuntura actual, requiere ineludiblemente hacerlo en diálogo con su devenir. Seguidamente, en otra nota preliminar, justificamos la elección de centrar el estudio en el nivel universitario. Avanzando más concretamente en la tesis que orienta el trabajo, proseguiremos a definir la discapacidad como "comunidad bivalente" en términos de Fraser (2000): exponiendo en qué medida abarca dimensiones económico-políticas y dimensiones de valoración cultural, proponemos la necesaria implicancia de redistribución y reconocimiento. Teniendo en cuenta este marco general, profundizaremos el análisis según 
las categorías de accesibilidad antes mencionadas. Concluimos planteando algunas reflexiones finales más no definitivas.

\subsection{Sinopsis histórica de la relación entre discapacidad y educación}

Históricamente, la discapacidad fue concebida como una cualidad de las personas, ya sea atribuida "al Mal, el diablo y el pecado" (Jacobo, 2012, p. 22) o fundamentada en definiciones médicas, ${ }^{2}$ que las volvía incapaces de participar en la vida social. En consecuencia, su educación estuvo fuertemente signada por la exclusión o la segregación en espacios creados al margen de los trayectos educativos "comunes", de carácter asistencialista, centrados en el cuidado, la compensación y la rehabilitación (Acuña y Bulit Goñi, 2010; Di Pietro y Pitton, 2012).

En contrapartida, las "Normas Uniformes sobre la igualdad de oportunidades para las personas con discapacidad" (ONU, 1994) y la "Declaración de Salamanca” (UNESCO, 1994) son considerados hitos normativos hacia la superación de dichas lógicas discriminatorias y segregacionistas al proclamar "la necesidad y urgencia de impartir enseñanza a todos los niños, jóvenes y adultos con necesidades educativas especiales dentro del sistema común de educación" (UNESCO, 1994, Art. viii). Así la "integración educativa" pasó a conformar el núcleo de un nuevo consenso sobre la educación de las personas con discapacidad (o con necesidades educativas especiales ${ }^{3}$ ) a nivel mundial.

Pero el traspaso de alumnos desde los trayectos de Educación Especial a los "comunes" fue un proceso "muy duramente criticado por haberse efectuado con escasos o nulos cambios en la escuela que acoge a esos alumnos, produciéndose lo que se ha tildado de simple integración física, no real" (Booth y Ainscow, 1998 en Parrilla Latas, 2002, p. 17). En otras palabras, más allá de ciertos avances a nivel normativo, político e incluso de consideración social (Acuña y Bulit Goñi, 2010), el mantenimiento de las estructuras institucionales y sus correspondientes nociones valorativas subyacentes naturalizadas,

\footnotetext{
2 Por ejemplo, en la Clasificación Internacional de Deficiencias, Discapacidades y Minusvalías (1980), se define a la discapacidad como "toda disminución (restricción) o ausencia (debido a una deficiencia) de la capacidad para realizar una actividad en la forma o dentro de un margen que se considera normal para un ser humano. La discapacidad sería así el resultado de la incidencia de una deficiencia que restringe o anula las habilidades de una persona para desarrollar una actividad considerada normal dentro de su contexto socio-cultural" (CIDDMM en Angelino, 2009, p. 44).

${ }^{3}$ Como se abordará más adelante, la propia denominación del colectivo no es unívoca ni "neutral", en el sentido de que es producto y al mismo tiempo produce una construcción cultural de la alteridad. En este caso se utilizan los términos "personas con discapacidad" y con "necesidades educativas especiales" en consonancia con su denominación en la normativa citada. Cuando este no sea el caso, se optará por el uso del término "personas discapacitadas".
} 
redundó en la "exclusión en el interior mismo de la escuela común" (Di Pietro y Pitton, 2012, p. 37).

Ante este panorama, nuevamente cristalizado en acuerdos internacionales como la Convención Interamericana para la Eliminación de todas las Formas de Discriminación Contra las Personas con Discapacidad (OEA, 1999) y la Convención Internacional sobre los Derechos de las Personas con Discapacidad (CDPCD) (2006), surgió un renovado consenso que aboga por la inclusión. Si bien esta no se circunscribe al ámbito educativo, sino que "constituye una idea transversal que ha de estar presente en todos los ámbitos de la vida (social, laboral, familiar, etc.)" (Parrilla Latas, 2002, p. 24), en este contexto "implica aquellos procesos que llevan a incrementar la participación de estudiantes, y reducir su exclusión del currículum común, la cultura y comunidad" (Booth y Ainscow, 1998, p. 2). Es decir, tal como sostiene Barton (1997):

...la educación inclusiva no es simplemente emplazar a los alumnos con discapacidades en el aula con sus compañeros no discapacitados; no es mantener a los alumnos en un sistema que permanece inalterado, no consiste en que profesores especialistas den respuestas a las necesidades de los alumnos en la escuela ordinaria (Parrilla Latas, 2002, p. 25).

Como anticipamos, una de las implicancias fundamentales de este reenfoque es la acentuación en la responsabilidad social por la discapacidad. Es decir, la discapacidad deja de ser una cualidad intrínseca y biológica de las personas para convertirse en una problemática social: "el eje de las acciones no está en las "personas con discapacidad" sino en el contexto social en el que ellas tienen derecho a desenvolverse" (Acuña y Bulit Goñi, 2010: 33). Precisamente por ello a este modelo se lo conoce como "Modelo Social de Discapacidad". Oponiéndose al modelo médico-céntrico centrado en la rehabilitación y normalización de las personas, "plantea la influencia social en el proceso que lleva a crear identidades discapacitadas, a través de una sociedad que es en sí misma discapacitadora (en su ambiente físico, en su política económica sanitaria, en su composición social) y que legitima una visión negativa de las diferencias" (Parrilla Latas, 2002, p. 20).

Sin lugar a dudas, el recorrido histórico hasta aquí descripto es sumamente sintético y simplificado, y responde casi exclusivamente a una periodización macropolítica. ${ }^{4}$ Como tal, incluso los lectores menos familiarizados con la problemática podrán advertir las distancias entre esta narración y la persistente yuxtaposición de concepciones y discursos en pugna manifiestos o menos conscientes-, y más aún respecto de experiencias y estadísticas que demuestran que las personas discapacitadas siguen siendo excluidas en los ámbitos

\footnotetext{
${ }^{4}$ Tomamos la definición de Narodowski (1996), quien propone: "Por periodización macropolítica se entiende el mecanismo por el cual las discontinuidades en el devenir histórico están representadas, en el discurso de la historia de la educación, por acontecimientos intrínsecos a los procesos políticos e institucionales operados en el más alto nivel gubernamental o estatal" (p.150). A propósito de ello, plantea que este tipo de lecturas suponen una correspondencia fuerte entre la política educativa y la práctica escolar, lo que denomina "falacia de covariancia".
} 
educativos (SIRIED, 2013). De modo que, más allá de que hacer un estudio histórico exhaustivo excedía ampliamente el propósito del trabajo, planteamos la síntesis de esta manera de forma intencional, precisamente para dar cuenta de dichas distancias.

Mientras no desconocemos la trascendencia de los avances que significó la emergencia del modelo social de discapacidad en los planos normativo y discursivo, interesa enfatizar que no resuelven el problema de justicia. Por el contrario, los proponemos como la puerta de entrada para abordar la cuestión. En este sentido, como hemos adelantado en la introducción, es necesario analizar los supuestos e implicancias de las metas que se proclaman en las políticas de inclusión educativa y las iniciativas concretas que se diseñan en respuesta. Nuestra hipótesis es que justamente parte del problema radica en cómo dichas metas y su concreción son entendidas desde una perspectiva filosófica-política, lo que nos trae nuevamente a considerar nuestro argumento central: retomando a Parrilla Latas (2002) si la educación inclusiva implica "cómo, dónde y por qué, y con qué consecuencias, educamos a todos los alumnos" (p. 25) darle respuesta a estos interrogantes únicamente desde el paradigma de la redistribución o del reconocimiento los resuelve solo parcialmente. Retomaremos esta discusión un poco más adelante.

\subsection{Sobre la relevancia de situar la discusión en el nivel universitario}

En Argentina, el nivel superior (que incluye el sistema universitario) no es obligatorio. Mientras tanto, hay claros indicios de que la educación básica obligatoria (que en el país se extiende desde la sala de 4 años hasta la culminación de la escuela secundaria -LEN, 2006) no está garantizada, sobre todo para determinados sectores de la población (incluyendo, pero no restrictiva a, los discapacitados). Teniendo en cuenta esto, más que en la literatura encontramos referentes que centran las discusiones sobre justicia educativa en visiones (más o menos) restrictivas de la educación, pero que por lo general no tienen alcance en el nivel superior (Ver, por ejemplo, Friedman, 1966), cabe atender la pregunta ¿por qué examinar la cuestión de la discapacidad en el nivel universitario en particular?

La respuesta más sencilla podría ser "porque está contemplado en la Ley". Es decir, la normativa que promulga el derecho al acceso a la educación para personas discapacitadas no se restringe a los niveles educativos obligatorios, sino que explícitamente incluye también el nivel superior. Efectivamente, en la CDPCD, adoptada por la República Argentina mediante la aprobación de la Ley 26.378 (2008), se dispone:

Los Estados Partes asegurarán que las personas con discapacidad tengan acceso general a la educación superior, la formación profesional, la educación para adultos y el aprendizaje durante toda la vida sin discriminación y en igualdad de condiciones con las demás. A tal fin, los Estados Partes asegurarán que se realicen ajustes razonables para las personas con discapacidad (Art. 24.5). 
Como hemos planteado antes, los términos en los que están formuladas normas como estas requieren ser analizados. ¿Qué quiere decir que las personas tengan acceso a la educación superior? ¿Qué implica el principio de no discriminación y la igualdad de condiciones? ¿Qué es un ajuste razonable, cómo se define, existe un límite a tal fin? Dirimir estas cuestiones exige desentrañar los supuestos, siempre en tensión, que se ponen en juego.

Esto cobra aún más relevancia si tenemos en cuenta que el sistema universitario tiene un rol central en la producción de conocimiento y en la formación de profesionales y ciudadanos, asociada al mismo tiempo al acceso a oportunidades laborales y de posiciones sociales ${ }^{5}$. En este sentido, podríamos cuestionar el argumento esgrimido por los defensores de la privatización de la educación superior (Friedman (1996) que proponen que hay una relación inversamente proporcional entre la especialización de las carreras y el beneficio público. Volveremos sobre esta cuestión más adelante, pero podemos anticipar algunos interrogantes que interpelan a dicha premisa: ¿Quiénes se beneficiarían si, por ejemplo, la formación docente contribuye a la adquisición de conocimientos y estrategias pedagógicodidácticas que contemplen la diversidad en las aulas? ¿Quiénes proyectan o construyen las rampas con la inclinación adecuada para transitar en silla de ruedas? ¿Cómo velar jurídicamente por los derechos de los discapacitados ante situaciones de discriminación? ¿Acaso contar con diseñadores industriales y programadores no favorece el desarrollo de tecnologías "adaptadas"? ¿Cómo deconstruir patrones culturales sin su problematización desde la producción académica, los medios de comunicación, las expresiones artísticas? Es decir, en los campos profesionales a los que hacemos referencia, como la formación docente, la ingeniería, el derecho, el diseño industrial, la programación, la comunicación, las artes, y otros tantos ejemplos que no presentaría un gran desafío seguir identificando, una formación que contemple el reconocimiento de la discapacidad podría representar un mayor beneficio público, particularmente para dichos sectores subalternizados.

Por otro lado, desde una perspectiva utilitarista hay quienes podrían cuestionar la dimensión de la problemática siendo que aproximadamente el $1 \%$ de la población universitaria es discapacitada (Misischia, 2018). Más en estos mismos términos, ¿no valdría considerar que entre la población con discapacidad casi el $87 \%$ no supera el secundario como máximo nivel educativo alcanzado (INDEC, 2018)?

Por lo expuesto, y aunque sin desconocer que se trata de un abordaje parcial de la cuestión pues la inclusión de personas discapacitadas no está garantizada en otros niveles educativos o ámbitos sociales, es que aquí se propone el foco en el nivel universitario.

\footnotetext{
${ }^{5} \mathrm{Si}$ bien esta es una premisa en sí misma cuestionable, examinarla en profundidad excede los límites de este trabajo. Por lo tanto, a estos fines, se tendrá en cuenta el estado de cosas respecto a la legitimación del sistema universitario en términos de la formación de profesionales y la producción de conocimiento.
} 


\section{La discapacidad como "comunidad bivalente"}

Centrar el análisis de la justicia educativa en un sector de la población en particular implica identificar la índole específica de la injusticia que afecta a ese grupo particular. Es decir, si se trata de una "injusticia socioeconómica, que está arraigada en la estructura económico-política de la sociedad" (Fraser, 2000, p. 27), o bien de tipo cultural o simbólica, "arraigada en los modelos sociales de la representación" (p. 28). No obstante, tal como advierte Fraser (2000), e incluso a pesar de que dicha distinción es fundamentalmente analítica, existen comunidades que especialmente se ven afectadas por ambos tipos de injusticia. Denomina a las mismas como "comunidades <<bivalentes>>" y las define en los siguientes términos:

...las comunidades bivalentes pueden ser víctimas tanto de una distribución socioeconómica desventajosa como de un reconocimiento cultural inadecuado, de forma que ninguna de estas injusticias es una consecuencia directa de la otra, sino que ambas son fundamentales y equivalentes en cuanto a sus causas. En este caso, ni las soluciones redistribuidas por sí mismas, ni las soluciones de reconocimiento por separado serán suficientes: las comunidades bivalentes precisan de ambas (p. 40).

Como anticipamos, la discapacidad tiene características que nos permiten definirla en estos términos. Considerando la dimensión económico-política, la discapacidad está fundada en relaciones sociales de producción de las cuales permanecen excluidos: “... la exclusión que produce la discapacidad es aquel movimiento que no admite la presencia de cierto tipo de personas con determinadas características en el espacio de la estructura económica" (Almeida, 2009, p. 218). En otras palabras:

La noción de discapacidad, tal como la concebimos, está fundada en las relaciones sociales de producción y en las demarcaciones que la idea de normalidad establece en estas sociedades modernas. En consecuencia, la visión trágica y mediatizada de este fenómeno es propia de la sociedad capitalista, producto de condiciones económicas, sociales y culturales: el cambio en la naturaleza del trabajo y del mercado de trabajo, las exigencias de la acumulación, la producción de sujetos útiles tanto a la producción como a la reproducción social y la tipología del hombre normal como sujeto deseable. Los "problemas" que acarrea la discapacidad son producciones sociales originadas en las relaciones de desigualdad social (Angelino, 2009, p. 49).

Desde esta perspectiva, en tanto injusticia distributiva, requiere medidas de redistribución. No obstante, como argumenta Young (1990), la justicia social no puede ser reducida a la distribución. En forma concomitante con este orden económico, existen procesos simbólicos y culturales que construyen una cierta valoración social sobre la discapacidad basada en una ideología de la normalidad. Es decir que la opresión que 
experimentan los miembros de grupos excluidos como los discapacitados es tanto producto de las estructuras de explotación como del imperialismo cultural. En este sentido,

La falta de reconocimiento vinculada a la discapacidad se instala y se transmite por medio de instituciones sociales que regulan la interacción social y que fundan sus discursos y prácticas en modelos de valores culturales que constituyen a ciertas categorías de actores sociales como normales y a otras como anormales. Esto trae consecuencias tan materiales como las que derivan de la distribución económica (Almeida, 2009, p. 223).

Así, en términos similares a cómo el androcentrismo o el racismo constituyen las bases de injusticias de reconocimiento en los casos de género y raza, el capacitismo "denota, en general, una actitud o discurso que devalúa la discapacidad, frente a la valoración positiva de la integridad corporal, la cual es equiparada a una supuesta condición esencial humana de normalidad" (Toboso, 2017: 1). Es decir se trata de la construcción cultural, "artificial" más normativa de la normalidad de los cuerpos. En la medida en que esto nos sitúa en el terreno de la valoración cultural, exige medidas de reconocimiento de la diferencia. Más específicamente, de una política que supere el principio de la igualdad de trato para reivindicar una autodefinición positiva de la diferencia (Young, 1990).

Claro que contemplar este paradigma no está exento de tensiones. De hecho, antes de seguir avanzando es necesario plantear una cuestión central: ¿son los discapacitados una comunidad? Y en tal caso, ¿cómo se define? En primer lugar, no puede ignorarse que, de manera similar a cuando se habla de la identidad de género o de raza, los colectivos son en sí mismos heterogéneos. En el caso de la discapacidad, esto es así tanto en los "tipos" de discapacidad (motriz, visual, auditiva, cognitiva, o combinaciones de varias, etc.) como incluso en distintas reivindicaciones identitarias al interior de estos (por ejemplo, entre sordos oralizados u no oralizados). Por otra parte, como hicimos referencia anteriormente, las definiciones de este sector de la población han variado históricamente, dando cuenta justamente de que son producto y productoras de una construcción cultural. Así, denominaciones como "personas con discapacidad" o "personas con necesidades educativas especiales" denotan su diferenciación en tanto individuos que tienen un déficit respecto del parámetro de normalidad. En cambio, en el marco del Modelo Social de discapacidad, se utiliza el término "discapacitados" bajo el supuesto de que es algo externo lo que los discapacita.

Dicho esto, "pensar a la discapacidad como una producción social, inscripta en los modos de producción y reproducción de una sociedad" (Angelino, 2009) exhibe nuevamente la necesidad de concebirla como producto de una valoración cultural, pero que no se da en el vacío, "sino en el marco de condiciones económicas que operan intrincadamente como texto y contexto del conflicto" (Almeida, 2009, p. 223). Por tanto, si se da tanto en función de 
una diferenciación económico-política como de valoración cultural, exige abordar la inclusión desde le paradigma de la justicia como redistribución y como reconocimiento. A continuación veremos ejemplos más concretos de qué quiere decir esta premisa.

\section{Discapacidad, redistribución y reconocimiento en el nivel universitario}

En las secciones que siguen profundizaremos el análisis sobre de qué modos y en qué medida la redistribución y el reconocimiento están implicados en las políticas universitarias de inclusión vinculadas a la discapacidad retomando, como se anticipó, los resultados de un estudio previo a modo de ejemplos ilustrativos. Para ello, tomamos como ejes tres categorías que nos permitirán hacer un examen más pormenorizado de la cuestión: (i) la accesibilidad formal, (ii) la accesibilidad académica, y (iii) la accesibilidad psico-sociocultural. Son categorías de uso frecuente en la literatura académica (Eroles y Fiamberti, 2011; Rositto, 2011; Rusler, 2006) y en las normativas, que consideramos apropiadas en la medida en que, consecuentemente con los postulados del Modelo Social, ponen el foco en la accesibilidad de los entornos (en contraposición a la integración de las personas). No obstante, como desarrollaremos en sus respectivas descripciones, sobre todo la accesibilidad formal (en algunos casos también asimilada como "accesibilidad edilicia") y la accesibilidad académica constituyen marcos para la acción frecuentemente asociados a la redistribución de recursos. Su uso en este contexto, entonces, es en cierta medida provocador: al analizar los supuestos en los que se sustentan, se busca poner en evidencia que tal como se entienden estrictamente resuelven solo parcialmente la cuestión.

\subsection{La accesibilidad formal}

La accesibilidad formal es quizás la forma más restrictiva en la que se entiende habitualmente la accesibilidad en general. Por un lado, implica la no discriminación en base a razones vinculadas a la discapacidad. En este caso, que las personas discapacitadas puedan ingresar a la universidad. En este sentido, como vimos, no existen restricciones declaradas para el ingreso al sistema universitario ( $\mathrm{y}$ de hecho la normativa vela por lo contrario). No obstante, si consideramos que sí es requisito la acreditación de la educación de nivel medio, y teniendo en cuenta que como advertimos aún persisten mecanismos de exclusión en los niveles inferiores del sistema educativo, se abren interrogantes en este sentido.

Más aún, cabe señalar que a pesar de que en la actualidad hay un compromiso discursivo con la igualdad y que las formas más explícitas de discriminación que imperaban hasta el Siglo XX cayeron en desuso, se podría argumentar que estas formas de opresión 
adquirieron una manifestación social diferente, pero siguen operando. De modo que, como mencionamos anteriormente, la no discriminación formal y la igualdad de trato (en este caso, que todos y todas puedan ingresar a la universidad) como principios fundamentales de justicia, son a todas luces insuficientes. Por el contrario, se hacen necesarias políticas de acción afirmativa, es decir, políticas de trato diferencial para grupos históricamente excluidos, que contribuyan a socavar la opresión (Young, 1990).

Un ejemplo de políticas de acción afirmativa paradigmático en el campo educativo son las becas dirigidas a grupos focalizados sistemáticamente excluidos. En Argentina, el sistema universitario de gestión pública es no arancelado, lo cual podría interpretarse nuevamente en el marco del principio de igualdad simple. No obstante, utilizamos el término "no arancelado" en oposición a "gratuito" para dar cuenta de que la extensión de las trayectorias educativas implica la postergación de la inserción en el mercado laboral (al menos a tiempo completo) y por tanto un coste de oportunidad de percibir ingresos, además de gastos asociados al transporte y a la compra de materiales de estudio, por ejemplo. Esto representa una condición de desventaja para ciertos sectores sociales para ingresar y permanecer en el sistema universitario, que además, teniendo en cuenta que el nivel educativo alcanzado incide en la distribución del trabajo y de ingresos, perpetua y reproduce la desigualdad. En este marco, las becas en general se presentan como políticas de redistribución asociadas a las injusticias socioeconómicas: a través de la distribución desigual de recursos se apunta a nivelar el punto de partida frente a la posibilidad de proseguir una carrera universitaria.

Efectivamente, existen en el país diversos programas de becas, como por ejemplo el Programa Nacional de Becas Universitarias (PNBU), que otorga un beneficio económico a estudiantes "de escasos recursos" (Resolución Ministerial N464/96). Pero, además, cuenta con subprogramas destinados a grupos específicos, como el subprograma para Alumnos Indígenas y, más relevante en el marco del presente trabajo, el subprograma para Alumnos Discapacitados.

Pero, como hemos propuesto al inicio, la discapacidad no puede ser definida únicamente en términos de desigualdad socioeconómica, razón por la cual políticas esencialmente redistributivas como las becas también resultan insuficientes. A este respecto cabe destacar que el mencionado subprograma para Alumnos Discapacitados del PNBU prevé ciertas excepciones en los requisitos necesarios para la acreditación y sostenimiento de las becas, como regímenes diferenciales de asistencia y de condición de regularidad. Esto nos introduce en el paradigma de la justicia como reconocimiento, en la medida en que (de manera intencional o no, pero podríamos interpretarlo así) implica identificar que dichos requisitos pretendidamente "objetivos" responden a parámetros de normalidad socialmente construidos, presentes de manera troncal pero al mismo tiempo casi imperceptible en las estructuras institucionales. 
En otras palabras, si desconocemos que los planes de estudio y los regímenes de asistencia están diseñados en función de un determinado perfil de alumno bajo criterios normativos y culturales, otorgarle una beca a una persona discapacitada para ingresar a la universidad y negarle su renovación al año siguiente porque aprobar la cantidad de materias previstas le requiere más tiempo de estudio o, por ejemplo, porque debe ausentarse para realizarse diálisis, es también un mecanismo de exclusión. Más aún, implica atribuirle la exclusión al excluido (Larrosa y Skliar, 2001), depositando el fracaso en el individuo, lo cual resulta doblemente violento. Por lo tanto, si tal como lo hemos definido el Modelo Social de discapacidad implica derribar las barreras sociales para la inclusión, este requerirá que las políticas redistributivas contemplen también las lógicas que subyacen a la asignación de recursos desde el paradigma del reconocimiento.

Otro tipo de medidas que frecuentemente se asocian con la accesibilidad formal son las que tienen que ver con la accesibilidad edilicia, que implica contar, justamente, con las condiciones edilicias (e.g. rampas, camineras, ascensores) y de equipamiento (e.g. señalética) necesarias para garantizar el ingreso y la libre circulación de las personas discapacitadas (especialmente discapacidades motrices y visuales) en las instituciones educativas. El Modelo Social generalmente se asocia fuertemente a la accesibilidad edilicia, quizás porque las barreras físicas son las más fácilmente identificables y para las cuales hay mayor desarrollo respecto a cómo sortearlas. Además, cabe señalar, las discapacidades motrices son las más predominantes en todos los niveles educativos, incluyendo el superior (INDEC, 2018).

A este respecto, vale mencionar la implementación del Programa de Accesibilidad en las Universidades Nacionales del Ministerio de Educación de la Nación, en el marco del cual se asignó financiamiento a las instituciones para la creación de "Circuitos Mínimos Accesibles" que "garanticen a todas las personas la posibilidad de acceso, circulación, permanencia y uso, a fin de desarrollar las actividades académicas y de apoyo específicas que se realicen en el edificio" (Res. N770, SPU, 2010). Se trata, entonces, de otro ejemplo de redistribución de recursos.

No obstante, la denominada "adecuación" de los espacios educativos y por tanto la asignación de recursos económicos a tal fin también responde a la preexistencia de criterios normativos y culturales sobre los cuerpos que pueden acceder y circular por dichos espacios. En este marco, resulta interesante señalar que mientras que en las universidades más longevas se hicieron necesarias tales adecuaciones, en la mayoría de los casos las que fueron creadas más recientemente las contemplaron desde su diseño inicial, hecho que podría representar un avance en tal sentido.

Otro aspecto interesante a considerar es que si bien la adecuación de los espacios está orientada a las personas discapacitadas en particular, se trata de una medida en cierta forma "anónima". Es decir, mientras la asignación de becas a la que anteriormente hicimos 
referencia implica la autoidentificación de los individuos como personas discapacitadas, algo que ha sido problematizado tanto teórica (ver, por ejemplo, Dubet, 2011) como empíricamente ${ }^{6}$, la accesibilidad edilicia responde al colectivo en general más allá de los individuos concretos (discapacitados o no) que hagan uso de las instalaciones.

Hasta aquí, entonces, hemos propuesto que la inclusión educativa de personas discapacitadas requiere de políticas específicas orientadas a tal fin, y comenzamos a exponer de qué maneras estas involucran tanto la redistribución como el reconocimiento, tarea que seguiremos profundizando a continuación.

\subsection{La accesibilidad académica}

Más allá de la accesibilidad formal, en la bibliografía que trata particularmente la inclusión educativa, también se hace alusión a la "accesibilidad comunicacional" (Eroles y Fiamberti, 2011) o "accesibilidad en relación a la información" (Acuña y Bulit Goñi, 2010). Es decir que existe un creciente consenso en contemplar que, sobre todo dada la especificidad de la educación en tanto actividad atravesada por la comunicación, además de las edilicias pueden presentarse otras barreras para la permanencia y eventual egreso de aquellas personas que tengan afecciones en los sentidos implicados. En líneas generales, tal como lo establece la CDPCD, la accesibilidad comunicacional implica "asegurar que la educación de las personas, y en particular los niños y las niñas [jóvenes y adultos] ciegos, sordos o sordociegos se imparta en los lenguajes y los modos y medios de comunicación más apropiados para cada persona y en entornos que permitan alcanzar su máximo desarrollo académico y social" (ONU, 2006, art. 24, inc. 3c). De esta manera, refiere a "todas las medidas pertinentes para que las personas con discapacidad puedan ejercer el derecho a la libertad de expresión y opinión, incluida la libertad de recabar, recibir y facilitar información e ideas en igualdad de condiciones con las demás y mediante cualquier forma de comunicación que elijan" (art. 21).

No obstante, en el marco del presente trabajo optamos por considerar la "accesibilidad académica", que va un paso más allá:

La accesibilidad académica integra, entonces, el principio de la accesibilidad universal a la vida universitaria y sin soslayar la obligada accesibilidad física y comunicacional (y el equipamiento que esto requiera) atiende específicamente a los aspectos curriculares, pedagógicos y didácticos para respaldar la formación integral, en función de los alcances de cada trayecto profesional particular y eso requiere que las casas de

\footnotetext{
${ }^{6}$ En un estudio previo (ver Luzuriaga, 2015) se definió como problemática la implementación de iniciativas como el relevamiento de personas discapacitadas que se llevó a cabo en 2012, pues los referentes entrevistados advertían que en muchos casos las personas discapacitadas no se identificaban como tales por miedo a la estigmatización. Profundizaremos este punto más adelante.
} 
estudio tengan la disposición necesaria para pensar respuestas singulares a situaciones igualmente singulares con anclaje en estos principios" (Acuerdo Plenario $\mathrm{N}^{\circ} 798 / 11$, p. 9).

En términos más concretos, la accesibilidad académica implica la distribución de recursos para, por mencionar algunos ejemplos, producir materiales bibliográficos en distintos soportes (audiolibros, textos en braille, etc.), equipar los salones de clase con tecnologías que favorezcan la acústica, la contratación de traductores en lenguaje de señas, etc. También este fue el caso de una extensión del Programa Conectar Igualdad, a partir del cual se distribuyeron gratuitamente computadoras a las personas discapacitadas que se encontraban cursando sus estudios universitarios, con el fin de que pudieran acceder a soportes tecnológicos para la traducción de materiales bibliográficos, toma de notas en distintos formatos, etc.

Sin por ello desconocer la legitimidad de dichas medidas, este eje de análisis nos introduce más profundamente en el paradigma del reconocimiento, en la medida requiere además la desnaturalización de ciertas normas culturales constitutivas de la gramática educativa. Para dar cuenta de ello citaremos a modo ilustrativo el caso de Emiliano, un estudiante de ingeniería civil que tiene parálisis cerebral. Tras entregar más de la mitad de su examen, que requería entre otras cosas el dibujo a mano alzada de una serie de diagramas y esquemas, en blanco, Emiliano desaprobó el examen parcial de la materia Hidráulica General. Tal como interpretó su profesor, esto podría significar que el estudiante no adquirió los aprendizajes necesarios para acreditar la asignatura. Pero esta lectura estaría desconociendo que la acreditación del aprendizaje a partir de dispositivos de evaluación en este, como en todos los casos, está mediada por determinados tipos de lenguaje y otras disposiciones arbitrarias, como el plazo de tiempo previsto para realizar la actividad. En palabras de Young (1990):

Los criterios de evaluación necesariamente conllevan implicaciones normativas y culturales y por tanto a menudo no serán neutrales respecto del grupo. Estos criterios a menudo conllevan presupuestos sobre los estilos de vida, estilos de conducta y valores que reflejan la experiencia de los grupos privilegiados que los diseñan e implementan. Dado que la ideología de la imparcialidad lleva a quienes evalúan a negar las particularidad de sus estándares, los grupos con experiencias, valores y formas de vida diferentes no satisfacen los criterios de la evaluación (p. 345).

En el caso de Emiliano, tener que dibujar esquemas en un tiempo acotado se le presentó como una barrera para dar cuenta de sus aprendizajes, dada su dificultad en la motricidad fina, hecho que se vio invisibilizado en la aparente imparcialidad de los criterios de evaluación. Por lo tanto, se hace evidente que la inclusión educativa implica el reconocimiento de tales diferencias. 
A este respecto, cabe hacer una mención especial a lo que se dispone en el acuerdo antes citado sobre la necesidad de "pensar respuestas singulares a situaciones igualmente singulares", lo que nos lleva a retomar la discusión sobre el reconocimiento colectivo y el posicionamiento de los individuos. En este sentido, podríamos decir que mientras el caso de Emiliano en particular se resolvió otorgándole más tiempo para realizar las evaluaciones y la posibilidad de hacerlo utilizando un dispositivo tecnológico, esto puede no ser suficiente o pertinente en otros. Esto nos lleva a considerar que abordar la problemática desde las individualidades resulta limitado en la medida en que podría llevar a velar el imperialismo cultural de grupos dominantes sobre otros, del mismo modo en que la autodefinición positiva de la diferencia de grupo debe atender a su propia diversidad intrínseca.

En la siguiente y última sección ahondaremos con mayor profundidad esta cuestión.

\subsection{La accesibilidad psico-socio-cultural}

Así como planteamos que en la educación, en tanto actividad atravesada por la comunicación, es imprescindible considerar las barreras de acceso a la información y académicas, podríamos agregar que en tanto actividad esencialmente social también hay que tener en cuenta lo que sucede en dichas relaciones, lo que se ha dado en llamar los "aspectos intersubjetivos" de la inclusión (Rusler, 2006) o "accesibilidad psico-socioculutural" (Rositto, 2011).

Es probablemente el eje más difícil de definir y de observar, pero al mismo tiempo (y quizás justamente por ello) crucial. Si bien, como ya hemos señalado antes, en la actualidad las formas más explícitas de discriminación y rechazo hacia la discapacidad se han restringido o al menos son moralmente cuestionadas, perduran en otras manifestaciones culturales y sociales. De hecho, en un informe sobre el estado de situación de las personas discapacitadas en la educación superior en nuestro país ya se advertía que

...los mayores obstáculos que enfrenta una persona con discapacidad en Argentina para acceder, permanecer y egresar de la educación superior están relacionados a las barreras actitudinales, a la percepción social de la discapacidad, dado que aún existen graves prejuicios que suscitan grandes conflictos en las relaciones interpersonales (Pugliese et al., 2005, p. 4).

Esto se condice con lo que señala Young (1990):

Gran parte de la experiencia opresiva del imperialismo cultural tiene lugar en contextos mundanos de interacción, en los gestos, el lenguaje, el tono de voz, y en la reacción de otras personas (cfr. Brittan y Maynard, 1984, págs.6-13). Pulsaciones de atracción y aversión modulan todas las interacciones, con consecuencias específicas para la experiencia del cuerpo. Cuando la cultura dominante define a algunos grupos como diferentes, como el <<otro>>, los miembros de esos grupos son atrapados en su cuerpo" (p. 209). 
El primer punto que nos interesa desarrollar en función de estas citas refiere a lo que ambas señalan como "relaciones interpersonales" o "interacción". Teniendo en cuenta, como describimos al comienzo del trabajo, que históricamente las personas discapacitadas han sido segregadas y excluidas de los ámbitos socialmente valorados, como las universidades, este no es un aspecto menor. En este sentido, es de destacar que los procesos de integración e inclusión incipientemente habilitan espacios de cohabitación, de encuentro. Es decir, comienzan a aparecer experiencias de "presencias" de aquellos <<otros>> cuerpos, que exigen, como expusimos hasta aquí, asumir responsabilidades respecto a cómo acceden a dichos espacios (Radi y Pérez, 2014).

No obstante, estas referencias a la interacción social no deben entenderse únicamente en términos del encuentro uno a uno, sino comprender que las experiencias opresivas mencionadas responden a pautas sociales y culturales profundamente arraigadas, casi inconscientes, vinculadas a la comovisión capacitista. En este marco, entendiendo a la justicia social como "la eliminación de la dominación y la opresión institucionalizadas" (Young, 1990: 32), la mera presencia física en condición excepcional y sin dar cuenta de dichos mecanismos de dominación y opresión, es igualmente violenta para los grupos subalternizados.

Parte de la deconstrucción de dichas visiones implica entonces hacerlas conscientes y problematizarlas. Para ello, retomando lo que anticipamos al inicio, las universidades pueden tener un rol fundamental por su incidencia en la legitimación de pautas culturales y profesionales. A este respecto, siguiendo las ideas de Radi y Pérez (2014), podríamos argumentar que de manera similar a lo que sucede con la "diversidad sexo-genérica", es preciso el abordaje de la "diversidad funcional" (en oposición a la idea capacitista). Por supuesto, cabe reconocer que en la actualidad hay iniciativas que avanzan en esta línea, adquiriendo la discapacidad una creciente presencia en publicaciones, iniciativas e incluso estructuras institucionales. Lo que aquí interesa advertir es que, en la medida en que la temática de la discapacidad aparezca en los planes de estudio o seminarios optativos desde los márgenes, sin una representación de la población tratada como "objeto de estudio" difícilmente constituyan políticas de reconocimiento, pues seguirán permaneciendo circunscriptas como excepciones dentro de una cultura predominantemente capacitista.

En relación a esto, un aspecto crítico es que, tal como hemos dado cuenta en este trabajo, las presencias que se encuentran (en mayor o menor medida) generalmente refieren a los estudiantes discapacitados. Una gran deuda en este sentido es contemplar qué sucede en los cuerpos docentes y espacios de tomas de decisiones. Es decir, ¿quién, cómo y desde qué lugar se habla de la discapacidad?

Por lo expuesto, el eje de la accesibilidad psico-socio-cultural nos remite más claramente al paradigma de la justicia del reconocimiento, pues pone en el centro de la discusión los espacios de construcción (o deconstrucción) de los "modelos sociales de 
representación, interpretación y comunicación" (Fraser, 2000) que subyacen a las injusticias culturales y simbólicas y por lo tanto intervienen en los otros ejes de análisis presentados.

\section{Reflexiones finales más no definitivas}

El análisis que hemos desarrollado remite, en esencia, a la pregunta por cuál paradigma de la justicia educativa, entendida como redistribución o reconocimiento, es más fértil para la inclusión de personas discapacitadas. En pocas palabras, reafirmamos la tesis inicial, sosteniendo que requiere acciones que se funden, de forma mancomunada y complementaria, en ambos paradigmas.

Teniendo en cuenta los principios del Modelo Social como la concepción de discapacidad de mayor consenso, promovida a nivel internacional y local, ahondamos en los supuestos implicados en las acciones que responden sobre sus tres ejes: la accesibilidad formal, la accesibilidad académica y la accesibilidad psico-socio-cultural. En los dos primeros casos se hizo más claramente visible la necesidad de contar con políticas de redistribución de recursos. Pero, al considerar que estas resuelven parcialmente la cuestión y pueden velar otros mecanismos de exclusión en tanto dejan inmutables las bases socioculturales e institucionales en las que se inscriben, se argumenta a favor de contemplar también el reconocimiento de la diferencia.

Por su parte, la accesibilidad psico-socio-cultural, eje central, importante no solo en sí mismo sino también por su clara incidencia en los otros ejes, remite más claramente al paradigma del reconocimiento. No obstante, tal como están las cosas en la actualidad, centrarse únicamente en esta dimensión podría significar desatender las desigualdades materiales que existen además de las simbólicas, limitando al mismo tiempo el reconocimiento. En términos más concretos, si entendemos la falta de reconocimiento como "estar expuesto/a a la invisibilidad en virtud de las prácticas de representación, comunicación e interpretación legitimadas por la propia cultura" (Fraser, 2000: 28) difícilmente podríamos avanzar hacia su eliminación si aquellos cuerpos son también invisibles (o ausentes) físicamente por faltas de recursos materiales.

Cabe hacer explícito que, en estos términos, aparece de alguna manera cierta idea de progresión: en la medida en que se transformen las bases culturales que legitiman las lógicas de exclusión e invisibilizan la diversidad, podrán hacerse menos necesarias políticas compensatorias. De un modo similar, esto podría llevar a sortear la innegable tensión que implica el reconocimiento de la diferencia sobre los individuos que deben reivindicar una identidad que se define en los mismos términos que los convierten en sujetos oprimidos. De ahí que de alguna manera el Modelo Social pueda entenderse como un estadio transicional hacia una transformación cultural que supere las lógicas binarias capacitistas 
normalidad/anormalidad, unos/otros, discapacitados/no discapacitados bajo la concepción de la diversidad funcional.

En relación con lo anterior, cabe retomar la idea de que la elección de situar el trabajo en el nivel universitario no fue arbitraria. Entendemos que es un ámbito que puede ser muy fructífero para iniciar el camino de transformación cultural no solo al interior mismo de las instituciones educativas, sino en todos aquellos ámbitos en los que se desenvuelvan los profesionales allí formados. No obstante lo cual, esto no va en detrimento de la necesidad de atender la problemática desde otros niveles y ámbitos educativos y sociales en general, y para ello esperamos que este trabajo aporte una posible matriz de análisis a recuperar.

Culminamos con una reflexión acerca de que entender la justicia educativa en los términos planteados necesariamente implica que sean trabajos como este, en los que se analizan iniciativas llevadas adelante desde la excepción, y desde una voz y mirada privilegiadas, los que operen desde los márgenes.

Agradecimientos: Agradezco a la Dra. Juliana Udi, quien motivó y orientó la realización de este trabajo.

\section{Referencias bibliográficas}

Acuña, C.H., y Bulit Goñi, L.G. (2010). Políticas sobre la discapacidad en la Argentina. El desafío de hacer realidad los derechos. Buenos Aires: Siglo Veintiuno editores.

Almeida, M.E. (2009). Exclusión y discapacidad: entre la redistribución y el reconocimiento. En Rosato, A. y Angelino, M. E. (Coords.) (2009) Discapacidad e ideología de la normalidad. Desnaturalizar el déficit. Buenos Aires: Noveduc.

Angelino, M. A. (2009). La discapacidad no existe, es una invención. De quienes fuimos (somos) siendo en el trabajo y la producción. En Rosato, A. y Angelino, M.E. (Coords.) (2009) Discapacidad e ideología de la normalidad. Desnaturalizar el déficit. Buenos Aires: Noveduc. Barton, L. (1997) Inclusive education: romantic, subversive or realistic? International Journal of Inclusive Education, 1(3), 231-242.

Bolívar, A. (2012). Justicia social y equidad escolar. Una revisión actual. Revista Internacional de Educación para la Justicia Social (RIEJS), 1(1), 9-45.

Booth, T., y Ainscow, M. (Eds.) (1998). From them to us: An international study of inclusion in education. Londres: Routledge.

Di Pietro, S. y Pitton, E. (Coords.) (2012). Las configuraciones de apoyo de la Dirección de Educación Especial en escuelas primarias comunes de gestión estatal. Estudio sobre los dispositivos que brindan orientación a las escuelas para el logro de trayectorias educativas integrales y el cumplimiento del os objetivos de inclusión educativa. Buenos Aires: Dirección 
de Investigación y Estadística, Ministerio de Educación del Gobierno de la Ciudad de Buenos Aires.

Fishkin, J.S. (1983). Justice, equal opportunity, and the family. Londres: Yale University Press.

Fraser, N. (2000). ¿De la distribución al reconocimiento? Dilemas de la justicia en la era post-socialista. En Butler, J., y Fraser, N. (2016). ¿Reconocimiento o redistribución? Un debate entre marxismo y feminismo. Madrid: New Left Review/ Traficantes de sueños. Fraser, N. y Honneth, A. (2005) ¿Redistribución o reconocimiento? Un debate político filosófico (pp. 23-66). Madrid: Morata.

Friedman, M. (1966). The Role of Government in Education. En Friedman, M. Capitalism and Freedom (pp. 85-100). Chicago y Londres: The University of Chicago Press.

Instituto Nacional de Estadística y Censos (INDEC) (2018). Estudio Nacional sobre el Perfil de las Personas con Discapacidad: resultados definitivos 2018. Ciudad Autónoma de Buenos Aires: Instituto Nacional de Estadística y Censos.

Jacobo, Z. (2012). Las paradojas de la integración/ exclusión en las prácticas educativas. Efectos de discriminación o lazo social. Buenos Aires: Centro de Publicaciones Educativas y Material Didáctico.

Luzuriaga, M. (2015). Iniciativas de accesibilidad e inclusión educativa para personas con discapacidades en Universidades Nacionales del Gran Buenos Aires. (Tesina de grado) Universidad de San Andrés, Buenos Aires, Argentina.

Misischia, B. S. (2018). La relación Universidad- Discapacidad. ¿Una inclusión excluyente? Educación, lenguaje y sociedad, 15(15). DOI: http://dx.doi.org/10.19137/els-2018-151506

Narodowski, M. (1996) La utilización de periodizaciones macropolíticas en Historia de la Educación. Algunos problemas. En Boom, A.M., y Narodowski, M. Escuela, historia y poder: miradas desde América Latina. Buenos Aires: Noveduc Libros.

Organización de los Estados Americanos (OEA) (1999). Convención Interamericana para la eliminación de todas las formas de discriminación contra las personas con discapacidad.

Organización de las Naciones Unidas (ONU). (1994). Normas Uniformes sobre la igualdad de oportunidades para las personas con discapacidad.

Organización de las Naciones Unidas (ONU). (2006) Convención Internacional sobre los Derechos de las Personas con Discapacidad.

Parrilla Latas, Á. (2002). Acerca del origen y sentido de la educación inclusiva. Revista de educación, 327, 11-29.

Pugliese, J.C., Técnico, E., Acquier, L.F., y Larrea, M. (2005). La integración de las personas con discapacidad en la educación superior en la República Argentina. Buenos Aires: Secretaría de Políticas Universitarias. 
Radi, B. y Pérez, M. (2014). Diversidad sexo-genérica en el ámbito educativo: ausencias, presencias y alternativas. XXI Jornadas sobre la enseñanza de la Filosofía. Coloquio Internacional 2014. Buenos Aires: Facultad de Filosofía y Letras.

Rawls, J. (1971). A Theory of Justice. Cambridge: Harvard University Press.

Rusler, V. (2006). Las perspectivas actuales y la proyección futura. En Prawda, A. y Rusler, V. ¿Por qué un Programa "Universidad y Discapacidad"? IV Jornadas Nacionales Universidad y Discapacidad. Facultad de Derecho de la Universidad de Buenos Aires, Buenos Aires, 5-7 de julio, 2006.

Toboso, M. (2017). Capacitismo. En Lucas Platero, R., Roson, M. y Ortega, E. (Eds.) Barbarismos queer y otras esdrújulas (pp. 73-81). Barcelona: Ed. Bellaterra.

UNESCO (1994) Declaración de Salamanca y Marco de Acción para las necesidades educativas especiales. Conferencia mundial sobre Necesidades Educativas Especiales: acceso y calidad. Salamanca, 7-10 de junio 1994. 